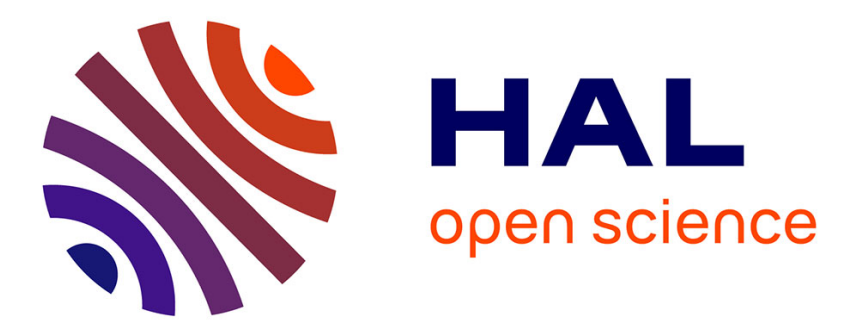

\title{
L'évolution de la vallée de la Marque dans le secteur de Bouvines (Nord, 59) depuis la fin du Pléniglacaire Weicshelien
}

\author{
Laurent Deschodt, Pierre-Gil Salvador
}

\section{To cite this version:}

Laurent Deschodt, Pierre-Gil Salvador. L'évolution de la vallée de la Marque dans le secteur de Bouvines (Nord, 59) depuis la fin du Pléniglacaire Weicshelien. Revue du Nord, 2009, Archéologie de la Picardie et du Nord de la France, 383 (91), pp.19-27. 10.3917/rdn.383.0019 . hal-02055104

\section{HAL Id: hal-02055104 https://hal-inrap.archives-ouvertes.fr/hal-02055104}

Submitted on 3 Mar 2019

HAL is a multi-disciplinary open access archive for the deposit and dissemination of scientific research documents, whether they are published or not. The documents may come from teaching and research institutions in France or abroad, or from public or private research centers.
L'archive ouverte pluridisciplinaire HAL, est destinée au dépôt et à la diffusion de documents scientifiques de niveau recherche, publiés ou non, émanant des établissements d'enseignement et de recherche français ou étrangers, des laboratoires publics ou privés. 


\section{L'évolution de la vallée de la Marque dans le secteur de Bouvines (Nord, 59) depuis la fin du Pléniglaciaire weichsélien}

Les plaines alluviales ont souvent constitué des espaces très attractifs pour les sociétés humaines, qu'il s'agisse de l'implantation des habitats ou du développement des activités économiques en relation avec l'eau. C'est pourquoi leur étude focalise, plus particulièrement depuis les années quatre-vingt en France, l'attention conjointe des archéologues et des spécialistes des archéosciences. L'étude des systèmes fluviaux a déjà montré, à différentes échelles spatiales et temporelles, leur grande sensibilité aux variations climatiques et à l'influence des hommes sur leur dynamique. Ces forçages ont pu conduire à une transformation sensible voire radicale des dynamiques hydrosédimentaires, se traduisant en autres effets, par la modification de la distribution des écoulements, avec pour incidences principales une évolution des paysages et des conditions de vie dans ces fonds de vallée. Ces observations ont fait l'objet de grandes synthèses à l'échelle du Nord-Ouest européen, qu'il s'agisse du domaine océanique ou continental ${ }^{1}$. Dans le Nord de la France, les travaux portant sur l'évolution des systèmes fluviaux et des fonds de vallée relèvent principalement des analyses synthétiques de J. Sommé 2 et plus récemment d'études plus ponctuelles liées à l'archéologie préventive ${ }^{3}$.

Un programme scientifique, engagé sous la direction de G. Leman-Delerive et porté par l'UMR 8164 HALMA-IPEL (Université Lille 3), vise depuis plusieurs années à une meilleure connaissance des

\footnotetext{
*. - Laurent Deschodt, INRAP Nord-Picardie, allée des champs, 59650 Villeneuve-d'Ascq CEDEX, UMR 8591 et visiteur UMR 8164 courriel: 1deschodt@nordnet.fr; Pierre-Gil SALVADOR, professeur des universités, Université Lille 1, UFR de Géographie et Aménagement, laboratoire TVES-EA4019 et visiteur UMR 8164, avenue Paul Langevin, 59655 Villeneuve-d'Ascq CEDEX, courriel: pierre-gil.salvador@univ-lille1.fr. Nous remercions Germaine Leman-Delerive (UMR 8164 HALMA-IPEL) qui a permis la réalisation de cette étude ainsi que
}

anthroposystèmes du bassin versant de la Deûle. Une première étude a porté sur le cours historique de cette rivière dans la région d'Houplin-Ancoisne ${ }^{4}$, couvrant une fenêtre chronologique depuis le Tardiglaciaire à l'Actuel. C'est dans ce cadre que de nouvelles investigations ont été menées sur la Marque, affluent principal de la Deûle, dont les principaux résultats font l'objet de cette présentation.

\section{DESCRIPTION DU SECTEUR D'ÉTUDE}

La Marque appartient au réseau de petites rivières qui parcourent le bas pays du Nord, région de plaines aux paysages composites se développant en contrebas du plateau de l'Artois. D'une longueur de $32 \mathrm{~km}$, elle draine un petit bassin versant de $217 \mathrm{~km}^{2}$ (fig. 1) qui s'étend sur les territoires du Pévèle au sud (point culminant à 106 m NGF), du Mélantois au centre et du Ferrain au nord où elle conflue en rive droite avec la Deûle (19 m NGF). Les plaines du Pévèle (substrat d'argile et de sable éocènes) et du Ferrain (sable et argile) présentent une topographie relativement variée pouvant apparaître sous l'aspect de basses collines, alors que le Mélantois constitue une plaine crayeuse aux ondulations presque insensibles ${ }^{5}$, les altitudes se distribuant entre 20 et $50 \mathrm{~m}$. Ces contrastes lithologiques infèrent sur le dessin de la vallée. Le franchissement de l'affleurement crayeux du Mélantois se réalise dans une vallée resserrée, alors que de part et d'autre du môle crayeux, la rivière a aménagé dans les

tous nos petits scarabées sondeurs

1. - BROWn 1997; MACKLIN et alii 2006; STARKEL 1983, 2006.

2. - SOMMÉ 1977.

3. - Deschodt 1999, 2002, 2009; Deschodt, Salvador 2003; DESCHODT et alii 2004; DESCHODT et alii 2005; DESCHODT et alii 2006. 4. - Deschodt, SAlvador 2003; Deschodt et alii 2004.

5. - Sommé 1977. 


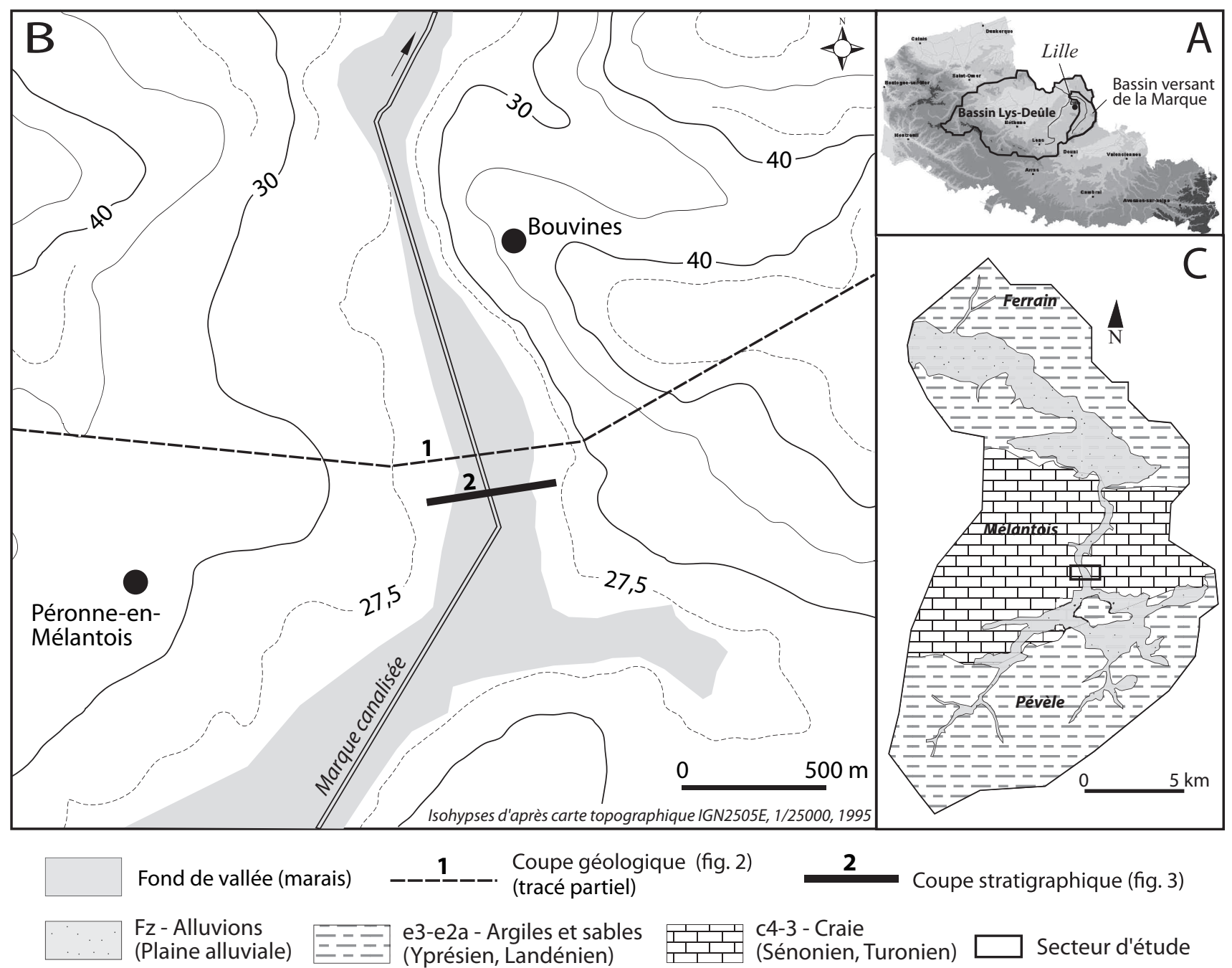

FIG. 1. - Localisation géographique (A, B) et contexte géologique $(C)$ du secteur d'étude.

formations tendres du Landénien deux vastes dépressions marécageuses. La pente de la Marque est faible, de l'ordre de $1,5 \%$ ò l'amont à moins de $0,5 \%$ oplus en aval dès l'entrée dans la première zone de marais. Le débit de la rivière est particulièrement modeste, $0,83 \mathrm{~m}^{3} / \mathrm{s}$ à Bouvines, près du secteur d'étude, et la partie aval de son cours est canalisée. Son régime hydrologique est de type océanique, avec des hautes eaux et des crues réparties sur la saison froide ${ }^{6}$.

Le secteur d'étude se situe dans la partie intermédiaire du bassin versant, près de la commune de Bouvines (mais sur le territoire de Cysoing) et à l'entrée de la section étroite de la vallée. Une coupe géologique simplifiée, réalisée à l'aide des sondages du

6. - FIEGel 1998.
BRGM, permet d'en apprécier la disposition (fig. 2). On peut mesurer la proximité du substrat crayeux séno-turonien de la surface, dont il n'est séparé pour l'essentiel que par un manteau de limons quaternaires dont la puissance n'excède guère $2 \mathrm{~m}$. La vallée s'inscrit dans la craie, une valeur approximative de son encaissement de l'ordre de $25 \mathrm{~m}$ pouvant être proposée. Le sommet du remblaiement alluvial forme, à une altitude voisine de $27 \mathrm{~m}$, une plaine d'environ $500 \mathrm{~m}$ de large. La proximité du substrat crayeux explique la présence locale de graviers de craie dans le remblaiement alluvial. J. Sommé ${ }^{7}$ montre que plus en aval, à la hauteur de la confluence avec la Deûle qui se trouve plus éloignée de la zone crayeuse, on ne trouve plus dans les sondages que des formations sableuses, ce

7. — SOMMÉ 1977. 


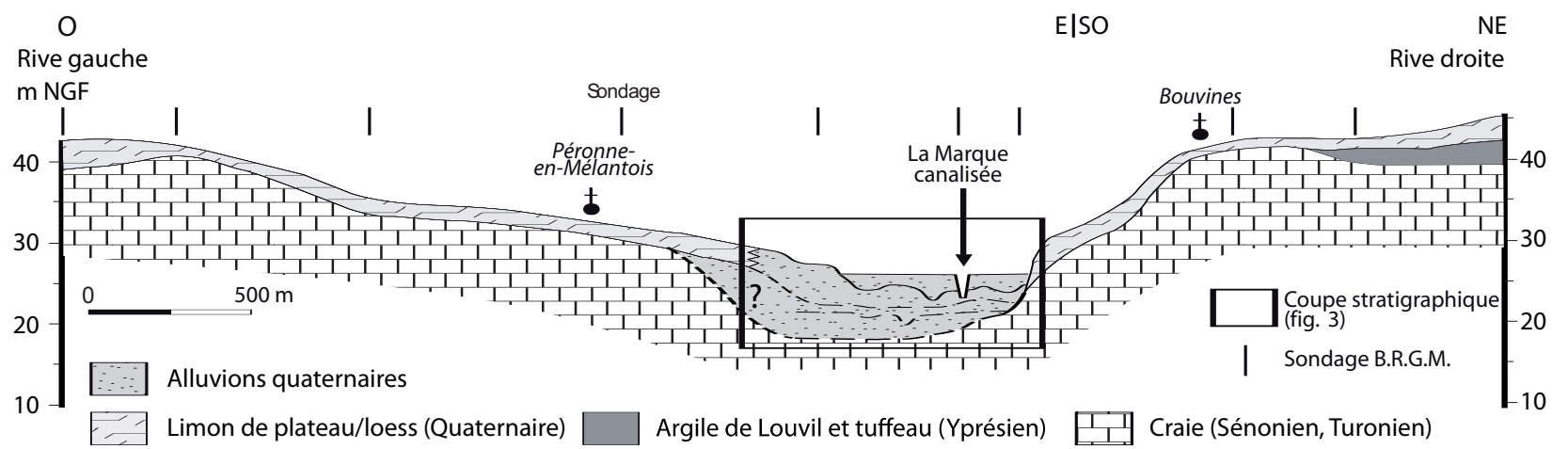

FIG. 2. - Coupe géologique simplifiée à travers la vallée de la Marque (secteur de Bouvines).

qui, précise l'auteur, rend difficile leur interprétation. On observera enfin la dissymétrie caractéristique des versants de la vallée, bilan d'une histoire paléogéographique régionale spécifique marquée par le dépôt en masse de limons éoliens durant les phases froides du Quaternaire. Soumis aux vents dominants d'ouest, le versant sous le vent est empâté par les limons (rive gauche) tandis que le versant au vent (rive droite) est plus escarpé et exposé à l'érosion.

L'intégralité de la vallée a été recoupée par une vingtaine de sondages réalisés à la tarière manuelle. Sans atteindre le substrat crayeux, ils ont permis de reconstituer la géométrie des formations fluvioéoliennes remblayant ce fond de vallée (fig. 3) et d'en restituer, à l'aide de datations radiocarbone, une partie de la chronologie. Après la description des unités lithostratigraphiques, une interprétation chronostratigraphique reconstituant l'évolution de ce secteur sera proposée.

\section{DESCRIPTION DES UNITÉS LITHOSTRATIGRAPHIQUES}

1. Craie: Elle est observée sur la remontée du versant de rive droite (S20), sous la forme de blocs de craie en fragments. Il s'agit probablement du toit de la craie blanche massive fragmentée attribuée au Sénonien, telle qu'elle est décrite dans le sondage du BRGM (20.4A.195) réalisé en fond de vallée. Ce niveau de base n'est pas atteint dans les autres sondages. Le toit de l'unité 1 constitue probablement le niveau d'arrêt des sondages S17 à S19.

2. Niveau d'altération du toit de la craie en place apparaissant sous la forme d'une pâte de craie blanche incluant des graviers de craie non roulés dans la masse (2a). Ce niveau pourrait être corrélé avec la craie jaune altérée décrite dans le sondage 20.4A.195 du BRGM (2b).

3. Niveau de sable moyen à grossier jaune-verdâtre dominant, devenant plus grossier à la base et s'enrichissant en granules de craie jusqu'à constituer une matrice de sable de craie incorporant des graviers non roulés et des fragments de craie au contact avec le niveau inférieur. Cette couche voit s'intercaler des niveaux plus fins de limon sableux à sable limoneux beige vert ou de limon plastique bleu légèrement sableux. L'ensemble du niveau pourrait être associé à un placage de sable tertiaire remanié sur versant.

4. Sous la terre arable (limon humifère graveleux) se développe un niveau de limon brun gris compact s'enrichissant progressivement en sable fin vers la base et incorporant dans toute sa masse de nombreux points de craie. Une limite de décarbonatation est observée à $198 \mathrm{~cm}$. Il pourrait s'agir d'un niveau de limon loessique pédogénisé sur le versant.

5. Formations fluviatiles (Pléniglaciaire weichsélien?). Il s'agit des alluvions constituant la nappe de fond de la Marque. Elle comprend des niveaux lités d'épaisseur variable pluri-centimétrique, formés de sables moyens à grossiers beige-gris à granules de craie épars, de graviers de craie (Ø5 mm) peu compactés et peu roulés, mais incluant également des niveaux de sédiments plus fins, sable fin à limon argileux beige à bleu. Le niveau n'est pas d'une grande homogénéité et voit alterner des secteurs où la sédimentation est plus grossière (S11/S8/S7/S5/S3/S2) ou plus fine (S12/S6/S4). 


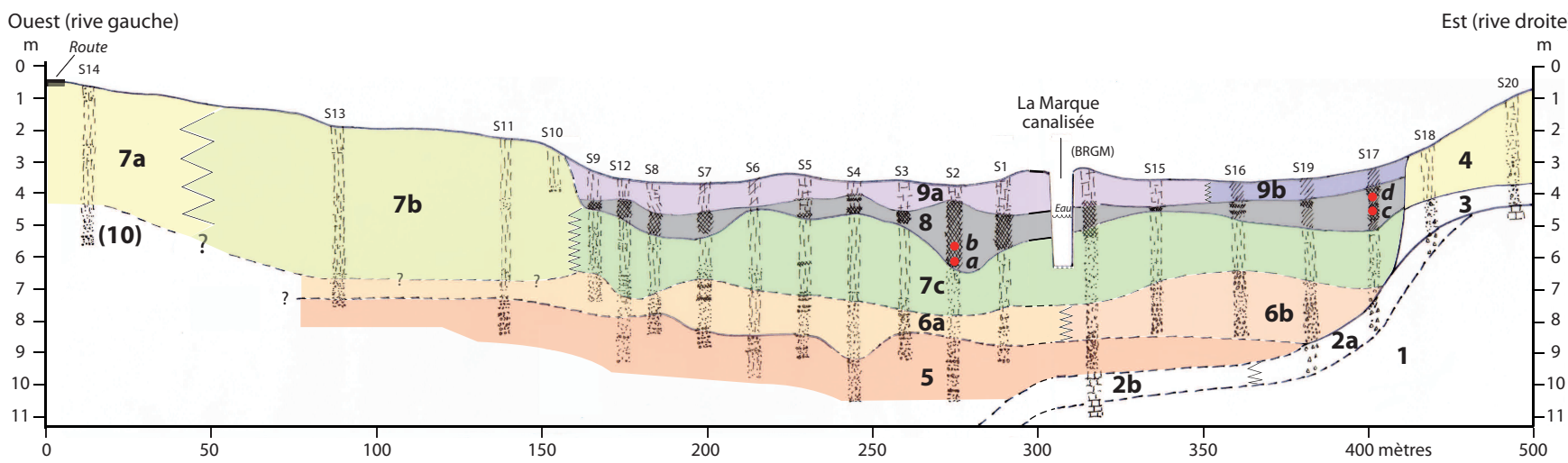

FIG. 3. - Coupe stratigraphique à travers le fond de vallée de la Marque.

Datations radiocarbone : $\boldsymbol{a}: 12115 \pm 90$ BP (12202-10838 av. J.-C.) (Ly-14092) ; $\boldsymbol{b}: 10685 \pm 60$ BP (10879-10708 av. J.-C.) (Ly-14091) ; $\boldsymbol{c}: 9720 \pm$ 50 BP (9278-8953 av. J.-C.) (Ly-14094) ; $\boldsymbol{d}: 4915 \pm 40$ BP (3772-3641 av. J.-C.) (Ly-14093).

Sondages: S1 à S20 : sondages manuels ; (BRGM) : sondage 20.4A.195 projeté sur la coupe et réalisé $40 \mathrm{~m}$ en amont par le BRGM (1954).

Unités lithostratigraphiques : 1. Craie ; 2. Pâte de craie blanche enrobant des graviers de craie non roulés (2a). Craie jaune altérée dans le sondage BRGM (2b) ; 3. Graviers de craie non roulés dans une matrice de sable de craie moyen à grossier de couleur crème et limon sableux beige verdâtre ; 4. Limon compact brun-gris à limon sableux beige vert à points de craie fréquents devenant progressivement plus sableux vers la base ; 5. Sable moyen à grossier beige riche en granules de craie dominant, entrecoupé de niveaux de graviers de craie peu roulés ; 6 . Sable fin à grossier gris beige avec graviers de craie épars souvent en litage avec des limons sableux à limons argileux gris beige (6a). À l'est le niveau devient plus grossier avec une alternance de graviers de craie anguleux et de sable moyen à grossier beige $(6 \mathrm{~b})$; 7. Limon brun jaune dominant (7a) passant à un limon gris bleu entrecoupé de petits lits de limons sableux (7b). Vers l'est, le niveau s'enrichit légèrement en sable avec des litages plus marqués (7c); 8. Limon tourbeux à tourbe limoneuse calcaire avec malacofaune abondante ; 9. Limon à limon argileux plastique brun à bleuté, souvent gleyifié (9a), devenant plus organique vers l'est $(9 \mathrm{~b})$; 10. Sable fin à moyen dominant gris à verdâtre en litages pluricentimétriques, avec des lits de graviers de craie vers la base.

6. Formations fluviatiles constituées d'un ensemble (6a) de niveaux lités d'épaisseur pluri-centimétrique voyant alterner des sables fins à moyens dominant, localement grossiers, gris-beige, incluant des granules de craie éparses, des sables limoneux à limons argilo-sableux beige à grisbeige. Des petits lits de graviers de craie (20$30 \%$ ) dans une matrice de sable grossier sont parfois observés (S7/S5/S3/S1). Ce niveau (6a) se distingue du précédent (5) par une granulométrie d'ensemble plus fine. Plus à l'est, il devient plus grossier (6b) et voit réapparaître en alternance des lits de sables moyens à grossiers beiges à graviers de craie épars et des lits pluri-centimétriques de graviers de craie anguleux (Ø2 $\mathrm{cm}$ maximum). Cet enrichissement en granules de craie peu usées traduit l'influence immédiate du versant crayeux de rive droite.

7. Sous les labours se développe un limon brun jaune non calcaire, riche en « points » de craie dispersés dans la masse du dépôt (7a). Ce niveau pourrait être assimilé à un limon éolien de type loess. Il laisse place plus à l'est à un limon gris dominant lité de petits lits sableux verts, alternant parfois avec un limon sableux verdâtre ou localement avec un limon plastique gris (7b). Le niveau devient plus sablonneux vers l'est (S11) et fran- chement lité en profondeur, avec une alternance de sables argileux et de limons argileux gris-bleu ou de limons beiges, d'épaisseur pluri-centimétrique. Cette unité peut être envisagée comme un dépôt issu du remaniement des limons éoliens voisins. Elle se prolonge dans le fond de vallée, où le remblaiement conserve un caractère lité plus ou moins marqué, alternant des niveaux de limon gris-bleu à beige, localement des limons argilosableux ou des limons sableux, avec des niveaux de sable limoneux à sable moyen gris-beige $(7 \mathrm{c})$.

8. Niveau à dominante organique, de type limon tourbeux ou limon organique gris-noir mou, compressible, à passages plus clairs avec malacofaunes souvent abondantes. Des niveaux centimétriques de limon clair tuffacé avec malacofaunes éparses s'intercalent localement dans ce dépôt, de même que des niveaux de sable fin et de limon plastique. Ce remblaiement organique a fait l'objet de quatre datations radiocarbone. Les deux premières sont réalisées dans la partie inférieure du dépôt et indiquent une mise en place durant la deuxième partie du Tardiglaciaire, depuis l'Allerød et jusqu'au Dryas récent: « a » $12115 \pm$ 90 BP (Ly-14092) et « b » $10685 \pm 60$ BP (Ly14091). La turfigenèse se poursuit durant la première partie de l'Holocène, depuis le Préboréal 


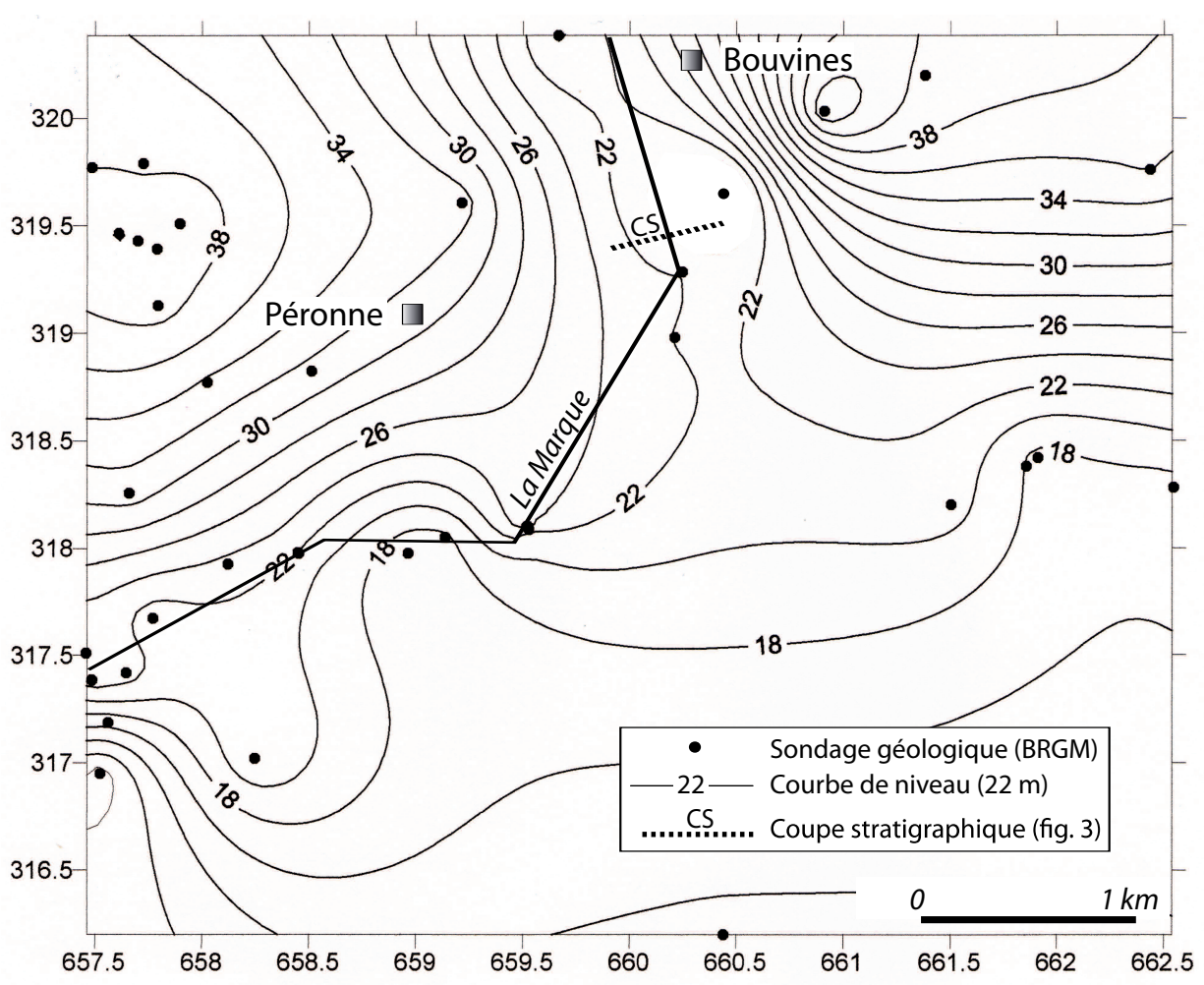

FIG. 4. - Paléotopographie du toit de la craie dans le secteur d'étude.

jusqu'à l'Atlantique récent: « $\mathrm{c}$ » $9720 \pm 50 \mathrm{BP}$ (Ly-14094) et « d » $4915 \pm 40$ BP (Ly-14093).

9. En surface se développe un limon humifère brun sombre à structure grumeleuse en rive gauche, riche en radicelles, devenant plus homogène et plastique vers le bas, de type gley (9a). Un fragment de tuile situé à $0,53 \mathrm{~m}$ sous la surface a été retrouvé dans cette unité (sondage S3). Plus à l'est, le niveau devient plus organique (9b).

10. Niveau de sable fin à moyen dominant, de couleur gris à verdâtre, organisé en litages pluricentimétriques dans lesquels s'intercalent parfois des niveaux plus limoneux. Des graviers de craie sont dispersés dans cette unité ou apparaissent vers la base en lits pluricentimétriques. Le niveau est difficile à corréler en raison de son altitude mais pourrait s'apparenter aux unités 5 et 6 .

\section{INTERPRÉTATION DES DONNÉES POUR UNE RECONSTITUTION DE L'ÉVOLUTION PALÉOGÉOGRA- PHIQUE DE LA VALLÉE}

\section{Le substrat crayeux}

Dans le secteur d'investigation, les formations superficielles reposent sur le toit de la craie du Sénonien, altérée sur plusieurs mètres d'épaisseur et décrite dans les sondages comme une craie jaune fissurée. Une paléotopographie du toit de la craie sénoturonienne, à l'échelle micro-régionale, est proposée (fig. 4), sur la base d'un MNT construit à l'aide de 44 sondages du BRGM. Le résultat est imprécis, eu égard au nombre insuffisant de points disponibles et à leur inégale répartition, mais il permet tout de même d'esquisser les grands traits de cette topographie. On distingue nettement la remontée du toit de la craie au nord et son affaissement vers le sud, où la craie est recouverte sur une vingtaine de mètres d'épaisseur par les formations argilo-sableuses du Landénien. Ce dispositif d'ensemble commande en surface la géométrie de la vallée de la Marque, largement évasée dans les formations éocènes et se contractant plus au nord au contact de la craie, comme l'illustre clairement la disposition des isohypses (fig. 4).

\section{Les nappes fluviatiles anciennes}

Elles sont assimilées aux unités 5 et 6 de la coupe, qui correspondent à la partie supérieure visible de la formation. La géométrie du remblaiement fluviatile n'est identifiée qu'en rive droite de la Marque, en raison de la proximité du toit de la craie. Ailleurs, le bed rock n'a pas été atteint. En se référant aux sondages relativement peu denses enregistrés au BRGM, nous pouvons extrapoler une épaisseur de dépôt de l'ordre 
de 4 à 5 mètres (la craie est atteinte dans le sondage 00204A0002/F1, $875 \mathrm{~m}$ en aval et sous la rive gauche, à 19,7 m NGF). Les faciès observés (litages de sables et limons, sable grossier à granules de craie) et l'absence de lit mineur distinct plaident pour une mise en place alluviale durant le Pléniglaciaire weichsélien. Ce faciès sableux à granules de craie homométriques, parfois sans matrice, est décrit par J. Sommé et L. Deschodt ${ }^{8}$ dans la vallée voisine de la Deûle lilloise. Il est considéré comme typiquement périglaciaire. La proximité du substrat crayeux altéré en rive droite de la Marque alimente le fond de vallée en fragments de craie mobilisés par le ruissellement (unité 6b). Ils sont alors déplacés sur de courtes distances, au vu du caractère anguleux ou de la faible usure des graviers de craie observés. Sur le secteur d'étude, la nappe fluviatile se perd sous le versant de rive gauche. Un sondage du BRGM (00204A0161/STM1), situé dans l'axe de la coupe, apporte un terminus à son extension (fig. 2) et implique, dans tous les cas, une nappe de moins d'un kilomètre de large.

\section{Les dépôts de versant et la nappe « fluvio- éolienne » du fond de vallée (unités $3,4,7$ )}

En rive droite, la craie est recouverte par un sable glauconieux à graviers de craie. Il est interprété comme un sable tertiaire remanié en position de versant. Des formations attribuées au Tertiaire sont observées sur le plateau, à proximité du versant, dans deux sondages géologiques, sous la forme d'une argile sableuse d'épaisseur métrique (BRGM00204B0003) ou, plus en retrait (BRGM00204X0220), une argile surmontée d'un « tuffeau bleu » (Landénien, argile de Louvil). Les deux versants de la vallée sont recouverts par un manteau de limon jaune quaternaire plus épais en rive gauche (4$5 \mathrm{~m})$ qu'en rive droite $(2-3 \mathrm{~m})$ et dont la partie supérieure est décalcifiée. La limite de décarbonatation est repérée vers $2 \mathrm{~m}$ de profondeur. Il s'agit probablement d'un loess. Toutefois, en rive gauche, le limon loessique devient de plus en plus lité en se rapprochant du fond de vallée. Il s'agit probablement d'un loess remanié par le ruissellement. On passe ainsi insensiblement, sans limite franche, d'un limon éolien non perturbé sur le versant (7a) au produit de sa remobilisation par les écoulements dans le fond de vallée (7c). Ce dispositif met en évidence la spécificité des vallées $\mathrm{du}$ bas pays dont le remblaiement doit beaucoup aux apports de versant. L'irrégularité et la faiblesse des

8. - Sommé 1977; Deschodt et alii 2004.

9. - SOMMÉ 1977.

10. - DESChODT et alii 2004; PraUd et alii 2007.

11. - VAndenberghe 1985; VAn Huissteden, VAndenberghe 1988 ; écoulements périglaciaires limitent le remaniement des formations accumulées, ce qui conduit à une géomorphologie fortement conditionnée par les influences locales ${ }^{9}$. D'après le même auteur, cet envahissement est déjà sensible durant la phase pléniweichsélienne moyenne (formations limonosableuses litées) et devient exclusive lors du dépôt du loess de couverture.

L'envahissement loessique du fond de vallée et le remaniement modéré du limon par les eaux caractérisent le bassin de la Deûle ${ }^{10}$. Le passage d'alluvions à des formations éoliennes ou fluvio-éoliennes est désormais bien connu dans les régions limitrophes en zone sableuse, du nord de la Belgique à l'Allemagne $\mathrm{du}$ Nord $^{11}$. On observe partout, à la fin du Pléniglaciaire supérieur, le dépôt d'un sable de couverture éolien qui scelle une sédimentation périglaciaire jusque-là à dominante fluviale. Selon Kasse ${ }^{12}$, ce phénomène était rendu possible par la dégradation du pergélisol qui favorisait par sa présence les écoulements superficiels. Nous proposons une corrélation entre le passage de l'unité 6 à l'unité 7 du transect de la Marque avec le passage des formations fluvioéoliennes à éoliennes dans les vallées de la zone sableuse, à la fin du Pléniglaciaire (datation postérieure à 17000 ans $^{13}$ ). En effet, le phénomène est identique si ce n'est qu'étant en zone limoneuse, ce n'est pas un sable de couverture mais un dépôt loessique plus ou moins lité qui s'accumule sous le vent. Quelques faibles écoulements dans le talweg peuvent expliquer la présence sporadique de lits sableux ou limoneux plastiques dans l'unité 7c. Ces mêmes écoulements permettent néanmoins une prise en charge d'une partie du limon puisque, en l'absence de recreusement ultérieur (cf. infra), ils constituent le seul processus susceptible d'expliquer, entre les sondages S9 et $\mathrm{S} 10$, le dénivelé du toit de l'unité 7 et la rupture de pente en limite de fond de vallée.

En l'absence de recreusement (cf. infra, description de l'unité 8), la topographie du toit de l'unité 7c est due aux ultimes écoulements périglaciaires. On dénombre deux ou trois axes d'écoulement sur le transect. Le mieux dessiné peut s'observer en rive gauche du canal actuel. Il constitue un petit chenal approximativement symétrique, aux bords convexes, de près de deux mètres de profondeur pour environ $90 \mathrm{~m}$ de large (de S4 à S15). Le second, qui se développe en bordure gauche du fond de vallée (S9 à S6), présente

VANDENBERGHe 1995; KASSE 1997; Mol 1997; HuISINK 2000; VAN HUISSTEDEN et alii 2000; KASSE et alii 2003; KASSE et alii 2007.

12. - KASSE 1997.

13. - KASSE et alii 2007. 
un profil en « $\mathrm{U}$ » aplati. Il est profond de moins d'un mètre pour une cinquantaine de mètres de large. Le troisième, encore moins marqué, occupe la bordure droite du fond de vallée (S15 à S17). Ces données morphométriques succinctes sont à rapprocher de celles faites sur un chenal observé à HouplinAncoisne, dans la vallée de la Deûle proche, et attribué à la même période: 2 à $3 \mathrm{~m}$ de profondeur pour 20 à $30 \mathrm{~m}$ de $\operatorname{large}^{14}$. On précisera toutefois que le méandre observé à Houplin-Ancoisne bénéficie d'un bassin versant un peu plus grand mais divague librement dans une large vallée tandis que les écoulements en aval de Bouvines sont confinés dans une vallée encaissée et plus étroite.

\section{L'enregistrement tardiglaciaire et holocène (unités 8 et 9)}

Il est représenté par un complexe limono-tourbeux d'une puissance variant de 1 à $3 \mathrm{~m}$, qui ennoie l'ensemble du fond de vallée. Il ne s'agit jamais d'une tourbe franche, mais plutôt d'un limon organique dans lequel s'intercalent des lits sableux ou limoneux, ce qui traduit une continuité des apports minéraux. Un niveau organique représente la base du dépôt tardiglaciaire-holocène (unité 8 ) et se développe de manière très irrégulière sur un à deux mètres d'épaisseur. Aucune trace de creusement ou dépôt grossier n'est observé à sa base. L'unité 8 ne semble qu'épouser la paléotopographie antérieure inscrite au sommet de l'unité 7 (cf. supra). Elle scelle et protège une morphologie héritée du Pléniglaciaire. L'ennoiement par la tourbe est l'expression d'un retour à des conditions plus tempérées et humides à la sortie du Pléniglaciaire, dans un contexte de secteur à faible pente favorable à la stagnation des eaux. Là encore, cette dynamique indique un environnement où l'indigence des écoulements ne permet pas le déblaiement des formations fluvio-éoliennes qui encombrent le fond de vallée. Il faut imaginer un lacis de chenaux, en zone humide, fonctionnant de manière épisodique, remblayés progressivement par la turfigenèse.

La datation la plus ancienne obtenue (sondage S2, fig. 3, $12115 \pm 90 \mathrm{BP} /$ Ly-14092) est attribuable à la fin du Bølling. Elle est précédée par $25 \mathrm{~cm}$ de dépôt calcaire tuffacé dont la mise en place au Pléniglaciaire parait improbable. Il peut par contre être rapproché de la sédimentation lacustre tuffacée observée dans le marais de Dourges (bassin versant de la Deûle) et daté d'environ 12230 à $12430 \mathrm{BP}^{15}$. Dans ce cas, les che-

14. - PRAUD et alii 2007.

15. - DESCHODT et alii 2005

16. - ANTOINE et alii 2000; PASTRE et alii 2000; ANTOINE et alii 2003. naux pléniglaciaires de la vallée de la Marque seraient le siège d'une sédimentation calcaire tuffacée, passant à de la tourbe au cours du Bølling. La même superposition tourbe sur tuf confirme le très faible écoulement dans le chenal.

La turfigenèse se poursuit assez curieusement durant l'épisode froid du Dryas récent (10685 \pm 60 BP/Ly-14091), plutôt caractérisé par une recrudescence d'apports sédimentaires ${ }^{16}$. On observera néanmoins sur la Marque que le remblaiement organique est subdivisé par deux niveaux de sable fin calcaire pluri-centimétriques, riches en malacofaune, qui encadrent le dépôt organique attribué au Dryas récent et peuvent indiquer de ce fait un regain des écoulements, signe de la dégradation climatique de cette période. Le faciès est d'ailleurs proche de celui observé dans la vallée de la Deûle pour la première partie du Dryas récent et avec des datations très proches (unité 9, dates vers $10410 \pm 60$ et $10570 \pm 60$ $\mathrm{BP}$, « unité 9: limon et limon sableux lité avec malacofaunes et débris végétaux $»^{17}$ ). Toutefois, contrairement à la vallée de la Deûle, nous n'observons pas de sédimentation limoneuse pour la seconde partie du Dryas récent. La bipartition du Dryas récent, avec une seconde période plus sèche, envisagée à HouplinAncoisne dans la vallée de la Deûle ${ }^{18}$ et par d'autres auteurs sur des bases paléontologiques ${ }^{19}$ se manifesterait donc dans la Marque par un arrêt de la sédimentation (seule la première partie du Dryas récent semble représentée).

La partie supérieure de l'accumulation organique se réalise à l'Holocène, qui voit se généraliser l'envahissement par la tourbe de la plupart des vallées du bas pays. La troisième date se positionne en effet dans le Préboréal (fig. 3, $9720 \pm 50$ BP/Ly-14094) et marque ainsi le début du réchauffement climatique holocène. Quant à la dernière datation, elle indique la fin de l'Atlantique récent (4915 \pm 40 BP/Ly-14093), dans la deuxième partie de l'Holocène. Elle est réalisée sur un limon organique situé à $0,50 \mathrm{~m}$ sur le précédent, ce qui illustre la très faible accumulation durant cette période de plus de 5000 ans. Comme sa partie inférieure, le dépôt organique est scindé en deux horizons par un niveau exclusivement minéral sous la forme d'un limon plastique gris. L'ensemble est recouvert par un limon plastique légèrement pédogénéisé en surface, d'épaisseur métrique. Ce sont les dépôts d'inondation de la Marque, aujourd'hui canalisée et individualisée en un chenal unique. Comme le

17. - DESCHODT et alii 2004.

18. - DESCHODT et alii 2004

19. — LIMONDIN-LOZOUET et alii 2002. 
souligne J. Sommé ${ }^{20}$, c'est l'intervention précoce des hommes sur le réseau hydrographique, progressivement chenalisé, qui conduit à son identification.

\section{CONCLUSION}

Le transect du fond de vallée de la Marque en aval de Bouvines révèle les traits marquants suivants :

1. Une forte dissymétrie morpho-stratigraphique avec le substrat crayeux sub-affleurant sur le versant est, au vent, et une forte épaisseur de limon sur le versant ouest, sous le vent.

2. Le limon présent en rive gauche (ouest) passe progressivement du loess à une formation fluvioéolienne en fond de vallée. Il recouvre une formation alluviale sableuse. Cette transition, marquée par le passage d'une alluvion à une couverture fluvio-éolienne avec processus éolien dominant, est caractéristique de l'aridification du climat à la fin du Pléniglaciaire.

3. La Marque est particulièrement peu active au Tardiglaciaire-Holocène. Elle utilise et nivelle la topographie héritée de la fin du Pléniglaciaire: des tufs puis des tourbes se déposent au Bølling et à l'Allerød dans les points bas hérités du Pléniglaciaire et le début du Dryas récent est enregistré sous la forme d'un sable lité avec une importante composante organique. Aucune incision ni écoulement important n'est enregistré à l'Holocène: les dépôts les plus compétents correspondent à des sables organiques datés du Dryas récent. Une formation tourbeuse envahit le fond de vallée dès le tout début de l'Holocène jusqu'à l'Atlantique. Ultérieurement, une alluvion un peu plus minérale (limon d'inondation) se superpose aux formations tourbeuses. On peut alors se demander si la vallée serait plus qu'une zone humide sans le canal anthropique qui la draine et si un lit mineur holocène a déjà existé.

Cette étude contribue à enrichir notre connaissance des dynamiques de fond de vallée régissant les rivières du Nord de la France depuis la fin du Pléniglaciaire weichsélien. On insistera sur l'intérêt que représente pour l'archéologue ce type de reconstitution paléogéographique, au service d'une meilleure compréhension des interrelations homme-milieu.

Mots-clés: Marque, fond de vallée, paléodynamique fluviale, Tardiglaciaire, Holocène.

\section{Bibliographie}

Antoine et alii 2000 : Antoine P., FAGnART J.-P., LimondinLozouet N., Munaut A.-V., « Le Tardiglaciaire du Bassin de la Somme: éléments de synthèse et nouvelles données », Quaternaire, 2000, 11 (2), p. 85-98.

Antoine et alii 2003: Antoine P., Auguste P., Bahain J.-J., Coudret P., Depaepe P., Fagnart J.-P., Falguères C., Fontugne M., Frechen M., Hatté C., Lamotte A., Laurent M., Limondin-Lozouet N., Locht J.-L., Mercier N., Moigne A.-M., Munaut A.-V., Ponel P., Rousseau D.-D., «Paléoenvironnements pléistocènes et peuplements paléolithiques dans la Somme (Nord de la France) », Bulletin de la Société préhistorique française, 2003, 100(1), p. 5-28.

Brown 1997: Brown A.G., Alluvial geoarchaeology. Floodplain archaeology and environmental change, Cambridge University Press, 1997, 377 p. (Cambridge Manuals in Archaeology)

DesChODT 1999 : DesChODT L., «Ébauche d'une cartographie au $1 / 10000^{\mathrm{e}}$ des formations superficielles de Lille. Synthèse sur la stratigraphie des sédiments de la vallée de la Deûle en contexte urbain, perçue à travers les fouilles archéologiques et les sondages géotechniques », Les Cahiers de la Préhistoire du Nord, 21 et 22, 1999.

DESCHODT 2002 : DesChODT L., « Une phase de sédimentation alluviale au Préboréal initial dans la vallée de l'Escaut (Nord de la France) », Quaternaire, 13, (2), 2002, p. 149-152.

DESChOdT 2009 : Deschodt L., «Quelques exemples de modification du tracé des rivières dans le Nord de la France », dans Revue du Nord, Hors-Série, coll. Art et Archéologie, 14, 2009, p. 19-27.

Deschodt, Salvador 2003 : Deschodt L., Salvador P.-G., « Contribution à la connaissance du paléoenvironnement de la vallée de la Deûle (Nord, France) : approche stratigraphique », Revue du Nord. Archéologie de la Picardie et du Nord de la France, t. 85, $\mathrm{n}^{\circ} 353,2003$, p. 23-33.

Deschodt et alii 2004 : Deschodt L., Salvador P.-G., Boulen M., « Formations sédimentaires et évolution de la vallée de la Deûle depuis le Pléniglaciaire supérieur à HouplinAncoisne (Nord de la France) », Quaternaire, 15, 3, 2004, p. 269-284.

Deschodt et alii 2005 : Deschodt L., Teheux E., Lantoine J., Auguste P., Limondin-Lozouet N., "L'enregistrement tardiglaciaire de Dourges (Nord de la France, bassin de la Deûle): Évolution d'une zone lacustre et gisements archéologiques associés », Quaternaire, 16 (3), 2005, p. 229-252.

Deschodt et alii 2006 : Deschodt L., Boulen M., CERCY Ch., Dessaux N., « Nouvelles données archéologiques sur la Deûle lilloise : d'une crise érosive du $\mathrm{II}^{\mathrm{e}} \mathrm{s}$. ap. J.-C. à l'urbanisation du lit mineur », Revue du Nord. Archéologie de la Picardie et du Nord de la France, t. 88, 2006, 368, p. 9-31.

FiEgEL 1998 : Fiegel M.-L., Atlas des zones inondables, vallée de la Marque, notice, DIREN Nord/Pas-de-Calais, 1998, $6 \mathrm{p}$.

Huisink 2000 : Huisink M., « Changing river styles in response to weichselian climate changes in the Vecht valley, eastern Netherlands », Sedimentary Geology, 2000, 133, p. 115134. 
KASSE 1997 : KASSE C., « Cold-climate aeolian sand-sheet formation in north-western Europe (c. 14-12.4 ka); a response to permafrost degradation and increased aridity », Permafrost and Periglacial Process, 1997, 8, p. 295-311.

KaSSE et alii 2003 : KASSE C., VANDENBERGHe J., VAN Hissteden J., Bohncke S. J. P., Bos J.A.A., « Sensitivity of Weichselian fluvial systems to climate change (Nochten mine, eastern Germany) », Quaternary Science Reviews, 2003, 22, p. 2141-2156.

KasSE et alii 2007 : Kasse C., VAndenberghe J., De Corte F., VAN DEN HaUte P., « Late Weichselian fluvio-aeolian sands and coversands of the type locality Grubbenvorst (southern Netherlands): sedimentary environnements, climate record and age », Journal of Quaternary Science, 2007, 22 (7), p. 695708.

LiMONDIN-Lozouet et alii 2002 : LIMONDIN-LOZOUET N., Bridault A., Leroyer C., Ponel Ph., Antoine P., Chaussé Ch., Munaut A.-V., Pastre J.-F., «Évolution des écosystèmes de fond de vallée en France septentrionale au cours du Tardiglaciaire : l'apport des indicateurs biologiques », dans BRAVARD J.-P., MAGNY M. (dir.), Les fleuves ont une histoire. Paléoenvironnement des rivières et des lacs français depuis 15000 ans, éditions Errance, 2002, p 45-62.

MACKLIN et alii 2006 : MACKLIN M.-G., BENITO G., GREGORY K.-J., Johnstone E., Lewin J., MichcZYnsKa D.-J., Soja R., STARKel L., ThORnDYCRAFT V.-R., « Past hydrological events reflected in the Holocene fluvial record of Europe », Catena, 66, 2006, p. 145-154.

MoL 1997 : Mol J., «Fluvial response to weichselian climate changes in the Niederlausitz (Germany) », Journal of Quaternary Science, 1997, 12 (1), 43-60.
Pastre et alii 2000 : Pastre J.-F., Leroyer C., LimondinLozouet N., Chausse Ch., Fontugne M., Gebhardt A., Hatte Ch., KRIER V., « Le Tardiglaciaire des fonds de vallée du Bassin parisien (France) », Quaternaire, 2000, 11, (2), p. 107122.

Praud 2007 : Praud Y. (dir.), Houplin-Ancoisne (59), Le marais de Santes, Rapport de fouille archéologique non publié $\mathrm{n}^{\circ}$ 02/0227/FOU, GB6115021001, Service régional de l'archéologie du Nord/Pas-de-Calais, 2 tomes, 2007, 290 pages, 279 figures.

Sommé 1977 : Sommé J., Les plaines du Nord de la France et leur bordure. Étude géomorphologique, thèse d'État, Université de Paris I, 1975, Paris, Champion éd., 1977, 2 vol.: p. 1-810, 185 fig.

Starkel 1983 : Starkel L., « The reflection of hydrologic changes in the fluvial environment of the temperate zone during the last 15000 years », dans GREGORY K. J. (éd.), Background to Palaeohydrology, Chichester, 1983, p. 213-235. STARKel et alii 2006 : StARKEL L., SoJA R., MichCZYNSKA D. J., « Past hydrological events reflected in Holocene history of Polish rivers », Catena, 66, 2006, p. 24-33.

VANDERBERGHE 1985 : VANDERBERGHE J., « Paleoenvironment and stratigraphy during the Last Glacial in the belgian-dutch border region », Quaternary Research, 24, 1985, p. 23-38.

VANDERBERGHe 1995 : VANDERBERGHE J., « Timescales, climate and river development ", Quaternary Science Reviews, 14,1995 , p. 631-638.

Van Huissteden, Vanderberghe 1988 : Van Huissteden J., VANDERBERGHE J., « Changing fluvial style of periglacial lowland rivers during the Weichselian Pleniglacial in the eastern Netherlands », Z. Geomorph. N.F., 1988, p. 131-146. 